\title{
Hermansky-Pudlak syndrome without pulmonary fibrosis
}

INSERM

\section{Source}

INSERM. (1999). Orphanet: an online rare disease and orphan drug data base.

Hermansky-Pudlak syndrome without pulmonary fibrosis. ORPHA:231512

Hermansky-Pudlak syndrome without pulmonary fibrosis as a complication includes three relatively mild types (HPS-3, HPS-5 and HPS-6) of Hermansky-Pudlak syndrome (HPS; see this term), a multi-system disorder characterized by ocular or oculocutaneous albinism, bleeding diathesis and, in some cases, granulomatous colitis. 